\title{
Broadband diffraction management and self-collimation of white light in photonic lattices
}

\author{
Ivan L. Garanovich, Andrey A. Sukhorukov, and Yuri S. Kivshar \\ Nonlinear Physics Centre and Centre for Ultra-high bandwidth Devices for Optical Systems (CUDOS), Research School of Physical \\ Sciences and Engineering, Australian National University, Canberra, ACT 0200, Australia
}

(Received 21 July 2006; published 21 December 2006)

\begin{abstract}
We introduce periodic photonic structures where the strength of diffraction can be managed in a very broad frequency range. We show how to design arrays of curved waveguides where light beams experience wavelength-independent normal, anomalous, or zero diffraction. Our results suggest opportunities for efficient self-collimation, focusing, and reshaping of beams produced by white-light and supercontinuum sources. We also predict a possibility of multicolor Talbot effect, which is not possible in free space or conventional photonic lattices.
\end{abstract}

DOI: 10.1103/PhysRevE.74.066609

PACS number(s): 42.25.Fx, 42.82.Et

It is known that periodic photonic structures can be employed to engineer and control the fundamental properties of light propagation $[1,2]$. In particular, the natural tendency of beams to broaden during propagation can be controlled through diffraction management [3,4]. Diffraction can be eliminated in periodic structures leading to self-collimation effect where the average beam width does not change over hundreds of free-space diffraction lengths [5]. On the other hand, diffraction can be made negative allowing for focusing of diverging beams [6] and imaging of objects with subwavelength resolution $[7,8]$.

The physics of periodic photonic structures is governed by scattering of waves from modulations of the refractive index and their subsequent interference. This is a resonant process, which is sensitive to both the frequency and propagation angle. Strong dependence of the beam refraction on the optical wavelength known as superprism effect was observed in photonic crystals [9]. Spatial beam diffraction also depends on the wavelength, and it was found in recent experiments $[5,10]$ that the effect of beam self-collimation is restricted to a spectral range of less than $10 \%$ of the central frequency. Such a strong dependence of the spatial beam dynamics on wavelength can be used for multiplexing and demultiplexing of signals in optical communication networks $[11,12]$. However, it remains an open question whether periodic photonic structures can also be used to perform an effective control of polychromatic and white-light beams such as those produced by light with supercontinuum frequency spectrum generated in photonic crystal fibers $[13,14]$.

In this paper, we demonstrate, for the first time to our knowledge, that intrinsic wavelength-dependence of diffraction strength in periodic systems can be compensated by geometrically induced dispersion and suggest periodic photonic structure designed for wavelength-independent diffraction management in a very broad frequency range covering up to $50 \%$ of the central frequency. We show the optimized periodic structures where multicolor beams experience constant normal, anomalous, or zero diffraction. This opens up opportunities for efficient self-collimation, focusing, and shaping of white-light beams and patterns.

We study propagation of beams emitted by a continuous white-light source in a periodic array of coupled optical waveguides [see Fig. 1(a)], where the waveguide axes are periodically curved in the propagation direction [see ex- amples in Figs. 2(a) and 3(a)]. Such waveguide array structures can be created using established fabrication techniques $[3,10]$. In the linear regime, the overall beam dynamics is defined by independent evolution of complex beam envelopes $E(x, z ; \lambda)$ at individual frequency components governed by the normalized paraxial equations,

$$
i \frac{\partial E}{\partial z}+\frac{z_{s} \lambda}{4 \pi n_{0} x_{s}^{2}} \frac{\partial^{2} E}{\partial x^{2}}+\frac{2 \pi z_{s}}{\lambda} \nu\left[x-x_{0}(z)\right] E=0,
$$

where $x$ and $z$ are the transverse and propagation coordinates normalized to the characteristic values $x_{s}=1 \mu \mathrm{m}$ and $z_{s}=1 \mathrm{~mm}$, respectively, $\lambda$ is the vacuum wavelength, $c$ is the speed of light, $n_{0}$ is the average refractive index of the medium, $\nu(x) \equiv \nu(x+d)$ is the refractive index modulated with the period $d$ in the transverse direction, and $x_{0}(z) \equiv x_{0}(z+L)$ defines the longitudinal bending profile of the waveguide axis with the period $L \gg d$. When the tilt of beams and waveguides at the input facet is less than the Bragg angle at each wavelength, the beam propagation is primarily characterized by coupling between the fundamental modes of the waveguides, and can be described by the tight-binding equations taking into account the periodic waveguide bending $[10,15], \quad i d \Psi_{n} / d z+C(\omega)\left[\Psi_{n+1}+\Psi_{n-1}\right]=\omega \ddot{x}_{0}(z) n \Psi_{n}$, where $\Psi_{n}(z ; \omega)$ are the mode amplitudes, $n$ is the waveguide number, $\omega=2 \pi n_{0} d / \lambda$ is the dimensionless frequency, and the dots stand for the derivatives. Coefficient $C(\omega)$ defines a coupling strength between the neighboring waveguides, and it characterizes diffraction in a straight waveguide array with $x_{0} \equiv 0[16,17]$. The coupling coefficient decreases at higher frequencies [18] and accordingly the beam broadening is substantially weaker at shorter wavelengths, see Figs. 1(b)-1(e).

We consider bending profiles which consist symmetric segments such that for each segment $x_{0}(z)=f\left(z-z_{a}\right)$ for a given coordinate shift $z_{a}$, where function $f(z)$ is symmetric, $f(z) \equiv f(-z)$. Then, after a full bending period $(z \rightarrow z+L)$ the beam diffraction is the same as in a straight waveguide array with the effective coupling coefficient $[10,15] C_{\mathrm{eff}}(\omega)$ $=C(\omega) L^{-1} \int_{0}^{L} \cos \left[\omega \dot{x}_{0}(\zeta)\right] d \zeta$. Therefore, diffraction of multicolor beams is defined by an interplay of bending-induced dispersion and frequency dependence of the coupling coefficient in a straight waveguide array. We suggest that spatial 

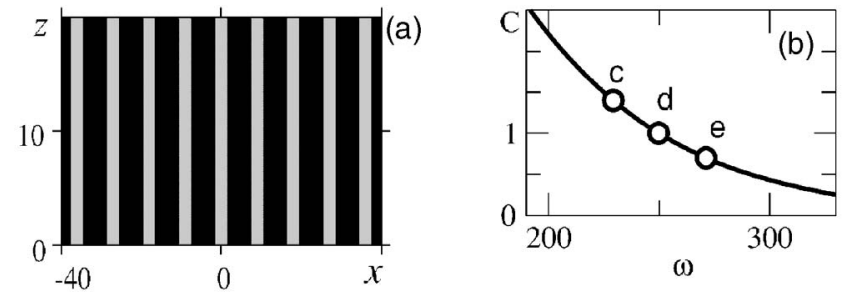

(a)

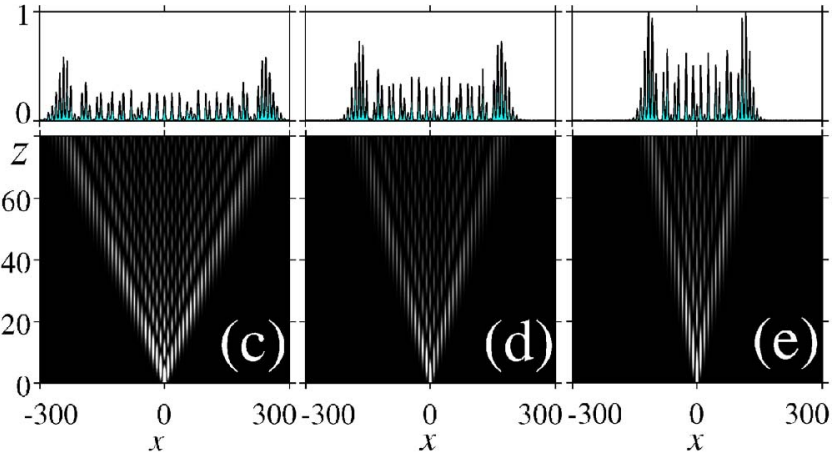

FIG. 1. (Color online) Discrete diffraction in (a) straight waveguide array with period $d=9 \mu \mathrm{m}$. (b) Coupling coefficient normalized to the coupling at the central frequency $C_{0}$. (c)-(e) Evolution of beam intensity and output intensity profiles after $80 \mathrm{~mm}$ propagation of a $3 \mu \mathrm{m}$ wide input beam for (c) $\lambda_{r}=580 \mathrm{~nm}$, (d) $\lambda_{0}=532 \mathrm{~nm}$, and (e) $\lambda_{b}=490 \mathrm{~nm}$, which correspond to the points "c, d, and e" in (b). Waveguide width is $3 \mu \mathrm{m}$ and substrate refractive index is $n_{0}=2.35$.

evolution of all frequency components can be synchronized allowing for shaping and steering of multicolor beams, when effective coupling remains constant around the central frequency $\omega_{0}$,

$$
d C_{\text {eff }}(\omega) /\left.d \omega\right|_{\omega=\omega_{0}}=0,
$$

and we demonstrate below that this condition can be satisfied by introducing special bending profiles.

First, we demonstrate the possibility for self-collimation of white-light beams, where all the wavelength components remain localized despite a nontrivial evolution in the photonic structure. Self-collimation regime is realized when the diffraction is suppressed and the effective coupling coefficient vanishes, $C_{\text {eff }}=0$. This effect was previously observed for monochromatic beams in arrays with zigzag [3] or sinusoidal [10] bending profiles, however, in such structures the condition of zero coupling cannot be satisfied simultaneously with Eq. (2), resulting in strong beam diffraction under frequency detuning by several percent [10]. We find that broadband diffraction management becomes possible in hybrid structures with a periodic bending profile that consists of alternating segments [see example in Fig. 2(a)], $x_{0}(z)=A_{1}\left\{\cos \left[2 \pi z / z_{0}\right]-1\right\}$ for $0 \leqslant z \leqslant z_{0}, x_{0}(z)$ $=A_{2}\left\{\cos \left[2 \pi\left(z-z_{0}\right) /\left(L / 2-z_{0}\right)\right]-1\right\}$ for $z_{0} \leqslant z \leqslant L / 2$, and $x_{0}(z)=-x_{0}(z-L / 2)$ for $L / 2 \leqslant z \leqslant L$. Effective coupling in the hybrid structure can be calculated analytically, $C_{\text {eff }}(\omega)$ $=C(\omega) 2 L^{-1}\left[z_{0} J_{0}\left(\xi_{1}\right)+\left(L / 2-z_{0}\right) J_{0}\left(\xi_{2}\right)\right]$, where $J_{m}$ is the Bessel function of the first kind of the order $m, \xi_{1}$ $=2 \pi A_{1} \omega / z_{0}$, and $\xi_{2}=2 \pi A_{2} \omega /\left(L / 2-z_{0}\right)$.

We select a class of symmetric profiles of the waveguide
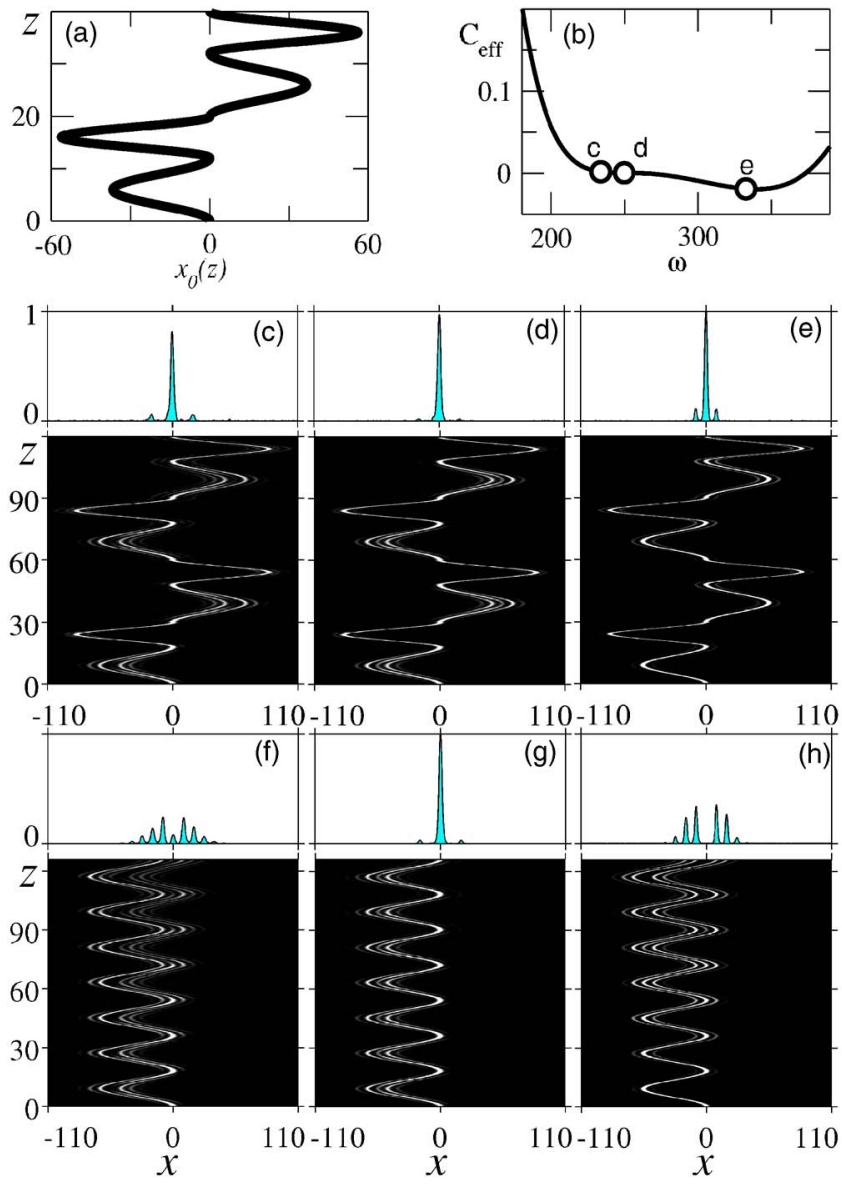

FIG. 2. (Color online) (a)-(e) Broadband self-collimation in an optimized waveguide array: (a) Waveguide bending profile with the period $L=60 \mathrm{~mm}$ and modulation parameters $A_{1}=27 \mu \mathrm{m}$, $A_{2}=42 \mu \mathrm{m}, z_{0}=18 \mathrm{~mm}$. (b) Effective coupling normalized to the coupling in the straight array at the central frequency $C_{0}=C\left(\omega_{0}\right)$. (c)-(e) Evolution of the beam intensity and output intensity profiles for different wavelengths marked (c) $\lambda_{r}=560 \mathrm{~nm}$, (d) $\lambda_{0}=532 \mathrm{~nm}$, and (e) $\lambda_{b}=400 \mathrm{~nm}$ corresponding to marked points in (b). (f)-(h) Frequency-sensitive diffraction in array with the sinusoidal bending profile at the wavelengths corresponding to plots (c)-(e).

bending to avoid asymmetric beam distortion due to higherorder effects such as third-order diffraction. Additionally, the waveguides are not tilted at the input, i.e., $\dot{x}_{0}(z=0)=0$, in order to suppress excitation of higher-order photonic bands by incident beams inclined by less than the Bragg angle. The effect of Zener tunneling to higher bands $[19,20]$ and associated scattering losses can be suppressed irrespective of the waveguide tilt inside the photonic structure by selecting sufficiently slow modulation to minimize the curvature $\ddot{x}_{0}(z)$ and thereby achieve adiabatic beam shaping.

In order to realize broadband self-collimation, we choose the structure parameters such that $\xi_{1}\left(\omega_{0}\right)=\widetilde{\xi}_{1} \simeq 2.40$ and $\xi_{2}\left(\omega_{0}\right)=\widetilde{\xi}_{2} \simeq 5.52$ are the first and the second roots of equation $J_{0}(\widetilde{\xi})=0$. Then, the self-collimation condition is exactly fulfilled at the central frequency $\omega_{0}, C_{\text {eff }}\left(\omega_{0}\right)=0$, and simultaneously the condition of frequency-independent coupling in Eq. (2) is satisfied for the following modulation parameters, $\quad A_{1}=\left[\widetilde{\xi}_{1} \widetilde{\xi}_{2} J_{1}\left(\widetilde{\xi}_{2}\right) / 2 \pi\left(\widetilde{\xi}_{2} J_{1}\left(\widetilde{\xi}_{2}\right)-\widetilde{\xi}_{1} J_{1}\left(\widetilde{\xi}_{1}\right)\right) \omega_{0}\right] L / 2$, 

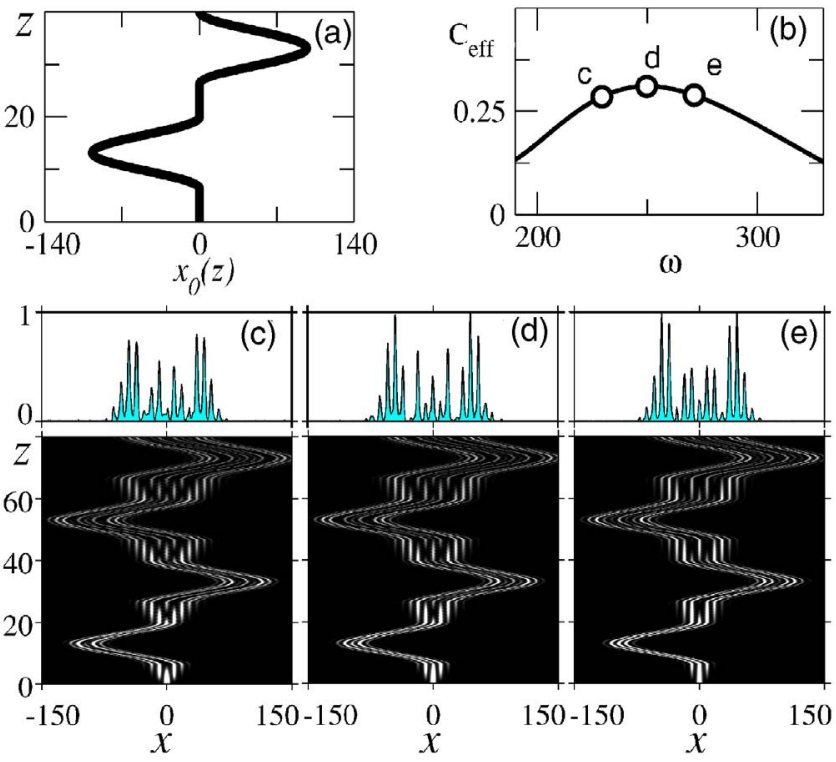

FIG. 3. (Color online) Wavelength-independent diffraction in an optimized periodically curved waveguide array. (a) Waveguide bending profile with the period $L=40 \mathrm{~mm}$ and (b) corresponding effective coupling normalized to the coupling in the straight array at the central frequency $C_{0}=C\left(\omega_{0}\right)$. (c)-(e) Evolution of beam intensity and output intensity profiles after propagation of two full periods for the wavelengths (c) $\lambda_{r}=580 \mathrm{~nm}$, (d) $\lambda_{0}=532 \mathrm{~nm}$, and (e) $\lambda_{b}=490 \mathrm{~nm}$, which correspond to the points $\mathrm{c}, \mathrm{d}$, and e in plot (b).

$A_{2}=-\left[J_{1}\left(\widetilde{\xi}_{1}\right) / J_{1}\left(\widetilde{\xi}_{2}\right)\right] A_{1}$, and $z_{0}=2 \pi \omega_{0} A_{1} / \widetilde{\xi}_{1}$. As a result, we obtain an extremely flat coupling curve shown in Fig. 2(b) where the point $\mathrm{d}$ corresponds to the central frequency. In this hybrid structure not only the first derivative vanishes according to Eq. (2), but the second derivative vanishes as well, $\left|d^{2} C_{\text {eff }}(\omega) / d \omega^{2}\right|_{\omega=\omega_{0}}|\sim| \widetilde{\xi}_{1} J_{2}\left(\widetilde{\xi}_{1}\right) J_{1}\left(\widetilde{\xi}_{2}\right)-\widetilde{\xi}_{2} J_{2}\left(\widetilde{\xi}_{2}\right) J_{1}\left(\widetilde{\xi}_{1}\right) \mid$ $<10^{-15}$. As a result, the effective coupling remains close to zero in a very broad spectral region of up to $50 \%$ of the central frequency. We note that the modulation period $L$ is a free parameter, and it can always be chosen sufficiently large to avoid scattering losses due to waveguide bending since the maximum waveguide curvature is inversely proportional to the period, $\max \left|\ddot{x}_{0}(z)\right| \sim L^{-1}$. Although the beam evolution inside the array does depend on the wavelength, the incident beam profile is exactly restored after a full modulation period, see examples in Figs. 2(c)-2(e), where results of numerical simulations of Eq. (1) are presented. Self-collimation is preserved even at the red (long-wavelength) spectral edge, where coupling length is the shortest and discrete diffraction in the straight array is the strongest [cf. Fig. 2(c) and Fig. 1(c)]. The hybrid structure provides a dramatic improvement in the bandwidth for self-collimation effect compared to the array with a simple sinusoidal modulation, where beams exhibit diffraction under small frequency detuning, see Figs. 2(f) $-2(\mathrm{~h})$.

We now analyze the conditions for frequency-independent normal or anomalous diffraction that may find applications for reshaping of multicolor beams. In order to reduce the device dimensions, it is desirable to increase the absolute value of the effective coupling and simultaneously satisfy
Eq. (2) to achieve broadband diffraction management. We find that Eq. (2) can be satisfied in the two-segment hybrid structure with $z_{0}=L / 2$ and $A_{1}=\left(\xi / 2 \pi \omega_{0}\right) L / 2$. Here a set of possible parameter values $\xi$ is determined from the relation $J_{0}(\xi) / J_{1}(\xi)=C_{0} \xi / C_{1} \omega_{0}$, where $C_{0}=C\left(\omega_{0}\right)$ and $C_{1}$ $=d C(\omega) /\left.d \omega\right|_{\omega=\omega_{0}}$ characterize dispersion of coupling in a straight array. It is possible to obtain both normal and anomalous diffraction regimes for normally incident beams, corresponding to positive and negative effective couplings $C_{\text {eff }}\left(\omega_{0}\right)=C_{0} J_{0}(\xi)$ depending on the chosen value of $\xi$. For example, for the waveguide array shown in Fig. 1, at the central frequency $\omega_{0}=250$ [corresponding wavelength is $\left.\lambda_{0}=532 \mathrm{~nm}\right]$ coupling parameters are $C_{0} \simeq 0.13 \mathrm{~mm}^{-1}$ and $C_{1} \simeq-0.0021 \mathrm{~mm}^{-1}$. Then, constant positive coupling around the central frequency $C_{\text {eff }}\left(\omega_{0}\right) \simeq 0.25 C_{0}$ is realized for $\xi \simeq 6.47$ and constant negative coupling $C_{\text {eff }}\left(\omega_{0}\right) \simeq-0.25 C_{0}$ for $\xi \simeq 2.97$.

We perform a comprehensive analytical and numerical analysis, and find that a hybrid structure with bending profile consisting of one straight (i.e., $A_{1} \equiv 0$ ) and one sinusoidal segment can provide considerably improved performance if $\omega_{0} C_{1} / C_{0}>\xi_{c r} J_{1}\left(\xi_{c r}\right) / J_{0}\left(\xi_{c r}\right)$, where value $\xi_{c r} \simeq 5.84$ is found from the equation $\left[J_{1}\left(\xi_{c r}\right)+\xi_{c r}\left[J_{0}\left(\xi_{c r}\right)\right.\right.$ $\left.\left.-J_{2}\left(\xi_{c r}\right)\right] / 2\right]\left[J_{0}\left(\xi_{c r}\right)-1\right]+\xi_{c r} J_{1}^{2}\left(\xi_{c r}\right)=0$. Under such conditions, larger values of positive effective coupling can be obtained in a hybrid structure with $A_{1} \equiv 0, A_{2}$ $=\left[C_{1} C_{\text {eff }}\left(\omega_{0}\right) / 2 \pi C_{0}^{2} J_{1}\left(\widetilde{\xi}_{2}\right)\right] L / 2, z_{0}=\left[C_{\text {eff }}\left(\omega_{0}\right) / C_{0}\right] L / 2$. In this structure, the effective coupling at central frequency is $C_{\text {eff }}\left(\omega_{0}\right)=\widetilde{\xi}_{2} C_{0}^{2} J_{1}\left(\widetilde{\xi}_{2}\right) /\left[\widetilde{\xi}_{2} C_{0} J_{1}\left(\widetilde{\xi}_{2}\right)+\omega_{0} C_{1}\right]$.

Example of a hybrid structure which provides strong wavelength-independent diffraction is shown in Fig. 3(a), and the corresponding effective coupling is plotted in Fig. 3 (b). The output diffraction profiles in this optimized structure are very similar in a broad spectral region, see examples for three wavelengths in Figs. 3(c)-3(e). We note that the outputs at these wavelengths are substantially different after the same propagation length in the straight waveguide array, as shown in Figs. 1(c)-1(e).

As one of the applications of the broadband diffraction

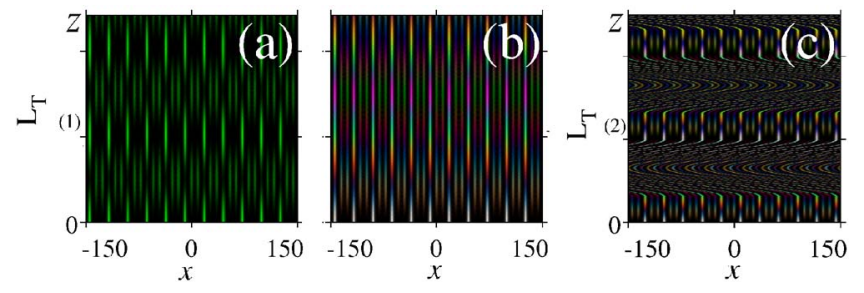

FIG. 4. (Color online) (a) Monochromatic Talbot effect in the straight waveguide array shown in Fig. 1(a): periodic intensity revivals every $L_{T}^{(1)}=16.5 \mathrm{~mm}$ of propagation for the input pattern $\{1$, $0,0,1,0,0, \ldots\}$ and the wavelength $\lambda_{0}=532 \mathrm{~nm}$. (b) Disappearance of the Talbot carpet in the straight array when input consists of three components with equal intensities and different wavelengths $\lambda_{r}=580 \mathrm{~nm}$ [redshifted], $\lambda_{0}=532 \mathrm{~nm}$ [green], and $\lambda_{b}=490 \mathrm{~nm}$ [blueshifted]. (c) Multicolor Talbot effect in the optimized structure with wavelength-independent diffraction [see Fig. 3.] Half of the bending period $L / 2=L_{T}^{(2)}=53.2 \mathrm{~mm}$ is equal to the Talbot distance for the corresponding effective coupling length. 
management we consider a multicolor Talbot effect which allows one to manipulate white-light patterns. The Talbot effect, when any periodical monochromatic light pattern reappears upon propagation at certain equally spaced distances, has been known since the famous discovery in 1836 [21]. It was recently shown that the Talbot effect is also possible in discrete systems for certain periodic input patterns [18]. For example, for the monochromatic periodic input pattern of the form $\{1,0,0,1,0,0, \ldots\}$, Talbot revivals take place at the distance $L_{T}^{(1)}=(2 \pi / 3)[1 / C(\omega)]$, see Fig. 4(a).

Period of the discrete Talbot effect in the waveguide array is inversely proportional to the coupling coefficient $C(\omega)$, which strongly depends on frequency, see Fig. 1(b). Therefore, for each specific frequency Talbot recurrences occur at different distances [18], and periodic intensity revivals disappear for the multicolor input, see Fig. 4(b). Multicolor Talbot effect is also not possible in free space where revival period is proportional to frequency. Most remarkably, multicolor Talbot effect can be observed in optimized waveguide arrays with wavelength-independent diffraction, see Fig. 4(c). In this example, we use the shape of structure with constant positive diffraction shown in Fig. 3, and choose half of the bending period to be equal to the period of the Talbot recurrences for the corresponding effective coupling in this structure, $L_{T}^{(2)}=(2 \pi / 3)\left[1 / C_{\text {eff }}(\omega)\right]$.

Similar ideas may also be applied to other fields where wave dynamics is governed by nonlinear Schrödinger equations (1) with $z$ standing for time. In particular, by introducing special periodic shift of lattice potential it may be possible to manipulate collectively multispecies Bose-Einstein condensates, where different wavelengths correspond to inverse masses of bosons from different species (e.g., [22]).

In conclusion, we have suggested periodic photonic structures where diffraction can be engineered in a very broad frequency range and light beams experience wavelengthindependent normal, anomalous, or zero diffraction. Our results suggest opportunities for efficient self-collimation, focusing, and shaping of polychromatic light beams and patterns. This may open up new possibilities for tailoring and enhancing nonlinear interactions of beams with different spectral content, which can be confined together for extended propagation distances.
[1] J. D. Joannopoulos, R. D. Meade, and J. N. Winn, Photonic Crystals: Molding the Flow of Light (Princeton University Press, Princeton, 1995).

[2] P. St. J. Russell, T. A. Birks, and F. D. Lloyd Lucas, in Confined Electrons and Photons, edited by E. Burstein and C. Weisbuch (Plenum, New York, 1995), pp. 585-633.

[3] H. S. Eisenberg, Y. Silberberg, R. Morandotti, and J. S. Aitchison, Phys. Rev. Lett. 85, 1863 (2000).

[4] Y. V. Kartashov et al., Opt. Express 13, 4244 (2005).

[5] P. T. Rakich et al., Nat. Mater. 5, 93 (2006).

[6] T. Pertsch, T. Zentgraf, U. Peschel, A. Brauer, and F. Lederer, Phys. Rev. Lett. 88, 093901 (2002).

[7] P. V. Parimi et al., Nature (London) 426, 404 (2003).

[8] Z. L. Lu et al., Phys. Rev. Lett. 95, 153901 (2005).

[9] H. Kosaka et al., J. Lightwave Technol. 17, 2032 (1999).

[10] S. Longhi, M. Marangoni, M. Lobino, R. Ramponi, P. Laporta, E. Cianci, and V. Foglietti, Phys. Rev. Lett. 96, 243901
(2006).

[11] L. J. Wu et al., IEEE J. Quantum Electron. 38, 915 (2002).

[12] J. Wan et al., Opt. Commun. 247, 353 (2005).

[13] J. K. Ranka et al., Opt. Lett. 25, 25 (2000).

[14] W. J. Wadsworth et al., J. Opt. Soc. Am. B 19, 2148 (2002).

[15] S. Longhi, Opt. Lett. 30, 2137 (2005).

[16] A. L. Jones, J. Opt. Soc. Am. 55, 261 (1965).

[17] S. Somekh et al., Appl. Phys. Lett. 22, 46 (1973).

[18] R. Iwanow et al., Phys. Rev. Lett. 95, 053902 (2005).

[19] C. Zener, Proc. R. Soc. London, Ser. A 145, 523 (1934).

[20] H. Trompeter, T. Pertsch, F. Lederer, D. Michaelis, U. Streppel, A Brauer, and U. Peschel, Phys. Rev. Lett. 96, 023901 (2006).

[21] H. F. Talbot, Philos. Mag. 9, 401 (1836).

[22] R. Pezer, H. Buljan, G. Bartal, M. Segev, and J. W. Fleischer, Phys. Rev. E 73, 056608 (2006). 